\title{
How to Generate Chaos from Switching System: A Saddle Focus of Index 1 and Heteroclinic Loop-Based Approach
}

\author{
Fang Bao and Simin Yu \\ College of Automation, Guangdong University of Technology, Guangzhou 510006, China \\ Correspondence should be addressed to Fang Bao, fainabao@126.com
}

Received 16 July 2011; Accepted 12 September 2011

Academic Editor: Reza Jazar

Copyright (c) 2011 F. Bao and S. Yu. This is an open access article distributed under the Creative Commons Attribution License, which permits unrestricted use, distribution, and reproduction in any medium, provided the original work is properly cited.

There exist two different types of equilibrium points in 3-D autonomous systems, named as saddle foci of index 1 and index 2, which are crucial for chaos generation. Although saddle foci of index 2 have been usually applied for creating double-scroll or double-wing chaotic attractors, saddle foci of index 1 are further considered for chaos generation in this paper. A novel approach for constructing chaotic systems is investigated by applying the switching control strategy and yielding a heteroclinic loop which connects two saddle foci of index 1. A basic 3-D linear system with an arbitrary normal direction of the eigenplane, possessing a saddle focus of index 1 whose corresponding eigenvalues satisfy the Shil'nikov inequality, is first introduced. Then a heteroclinic loop connecting two saddle foci of index 1 will be formed by applying the switching control strategy to the basic 3-D linear system. The heteroclinic loop consists of an unstable manifold, a stable manifold, and a heteroclinic point. Under the necessary conditions for forming the heteroclinic loop, the intended two-segmented piecewise linear system which exhibits the chaotic behavior in the sense of the Smale horseshoe can be finally constructed. An illustrative example is given, confirming the effectiveness of the proposed method.

\section{Introduction}

It is well known that saddle foci of index 2 are crucial for chaos generation in 3-D autonomous systems, where each saddle focus of index 2 creates one corresponding scroll or wing. Typical examples include the double-scroll Chua system, the double-wing Lorenz system, and so forth [1-5]. Among the three equilibrium points possessed by the double scroll Chua system, two are the saddle foci of index 2 with eigenvalues $\gamma, \sigma \pm j \omega$ satisfying $\gamma<0, \sigma>0$ and $|\sigma / \gamma|<1$, and one is the saddle focus of index 1 with eigenvalues $\gamma, \sigma \pm j \omega$ satisfying $\gamma>0, \sigma<0$, and $|\sigma / \gamma|<1$. The two saddle foci of index 2 create two corresponding 
scrolls leading to the emergence of the double-scroll chaotic attractor [1]. Among the three equilibrium points possessed by the double-wing Lorenz system, two are the saddle foci of index 2 with eigenvalues $\gamma, \sigma \pm j \omega$ satisfying $\gamma<0, \sigma>0$, and $|\sigma / \gamma|<1$, and one is the saddle point with eigenvalues $\gamma_{1}, \gamma_{2}, \gamma_{3}$ satisfying $\gamma_{1}<0, \gamma_{2}>0$, and $\gamma_{3}<0$. The two saddle foci of index 2 create two corresponding wings leading to the emergence of the double-wing chaotic attractor [2]. Similarly in many other double-scroll systems or the double-wing generalized Lorenz system family, two saddle foci of index 2 generate two corresponding wings or two scrolls [6]. Multiscroll or multiwing chaotic attractors can also be further generated by extending the number of saddle foci of index 2 [7-9].

The above-mentioned method is also applied for constructing switching systems, where only saddle foci of index 2 are considered for generating scrolls [10, 11]. For example, $\mathrm{Li}$ and Chen present an approach for constructing a piecewise linear chaotic system based on the heteroclinic Shil'nikov theorem, starting from a linear system with a saddle focus of index 2 [10]. A heteroclinic loop can be easily constituted by the proposed method; however it cannot be extended to the general case for the eigenplane of the linear system can only be located on the $x-y$ plane. In [11], a piecewise linear chaotic system is constructed based on heteroclinic Shil'nikov theorem and the switching control strategy, by particularly selecting two linear systems with two saddle foci of index 2, the eigenplanes of which are supposed to be symmetric. The method presented in [11] cannot be applied into the general situation due to the orientation restriction on the linear systems.

The review above indicates that for both smooth continuous chaotic systems and piecewise linear chaotic ones, saddle foci of index 2 have been usually considered for creating chaotic attractors. One may ask whether or not there is a possible way to break such a limitation? This paper will provide a positive answer to the question.

Based on the heteroclinic Shil'nikov theorem and the switching control strategy, this paper proposes a novel approach for constructing piecewise linear chaotic systems by employing saddle foci of index 1 . A basic 3-D linear system with an arbitrary normal direction of the eigenplane is first introduced. This 3-D linear system has a saddle focus of index 1 whose corresponding eigenvalues $\gamma, \sigma \pm j \omega$ satisfy $\gamma>0, \sigma<0$, and $|\sigma / \gamma|<1$. The necessary parameter conditions, which ensure the existence of the heteroclinic loop connecting two saddle foci of index 1 in the controlled switching system, are then deduced. In detail, the heteroclinic loop presented in the controlled switching chaotic system with saddle foci of index 1 consists of three geometric invariant subsets, which are a 1-D unstable manifold corresponding to real eigenvalues $\gamma$ satisfying $\gamma>0$, a 2-D stable manifold corresponding to complex conjugate eigenvalues $\sigma \pm j \omega$ satisfying $\sigma<0$ and $|\sigma / \gamma|<1$, and a heteroclinic point. Under the necessary parameter conditions for forming the heteroclinic loop, the intended 2-segmented piecewise linear system, which exhibits the chaotic behavior in the sense of the Smale horseshoe, can be finally constructed. The main differences between this presented method and $[10,11]$ lie in the following aspects: (i) two distinct types of saddle foci are considered for chaos generation, of which saddle foci of index 1 are considered in this paper while saddle foci of index 2 in $[10,11]$; (ii) the normal direction of eigenplane proposed in this paper is arbitrarily orientated while the eigenplane of the selected linear system can only be located on the $x-y$ plane in [10] or the orientations of the two linear systems are also restricted since their eigenplanes are supposed to be symmetric in [11].

The rest of the paper is organized as follows. A basic 3-D linear system with a saddle focus of index 1 is introduced in Section 2. Based on the heteroclinic Shil'nikov theorem and the switching control strategy, the intended 2-segmented piecewise linear system which exhibits the chaotic behavior in the sense of the Smale horseshoe is constructed in Section 3. 
The basic dynamic behaviors of the piecewise-linear chaotic system are further analyzed in Section 4. Finally, concluding remarks are given in Section 5.

\section{A Basic 3-D Linear Nominal System with a Saddle Focus of Index 1}

In this section, the heteroclinic Shil'nikov theorem is first described. A basic 3-D linear system, possessing a saddle focus of index 1 whose eigenvalues satisfy the Shil'nikov inequality, is then introduced. After that, the mathematical expressions of the corresponding eigenvectors, the unstable, and stable manifolds are derived.

\subsection{The Heteroclinic Shil'nikov Theorem}

Consider a 3-D autonomous dynamical system [12]

$$
\dot{x}(t)=\xi(x), \quad t \in \mathbf{R}, \quad x \in \mathbf{R}^{3},
$$

where the vector field $\xi: \mathbf{R}^{3} \rightarrow \mathbf{R}^{3}$. Let $P_{1}\left(x_{1}, y_{1}, z_{1}\right)$ and $P_{2}\left(x_{2}, y_{2}, z_{2}\right)$ be two distinct equilibrium points for (2.1). Suppose the following:

(i) both $P_{1}$ and $P_{2}$ are saddle foci that satisfy the Shil'nikov inequality $\left|\gamma_{i}\right|>\left|\sigma_{i}\right|>0$ (i= $1,2)$ with the further constraint $\omega_{i} \neq 0, \sigma_{1} \sigma_{2}>0$ and $\gamma_{1} \gamma_{2}>0$;

(ii) there is a heteroclinic loop $H_{l}$ joining $P_{1}$ to $P_{2}$ that is made up of two heteroclinic orbits $H_{i}(i=1,2)$.

Then both the original system of (2.1) and its perturbed varieties exhibit the Smale horseshoe chaos.

\subsection{Design of a Basic 3-D Linear Nominal System with a Saddle Focus of Index 1}

The state equation of the basic 3-D linear nominal system to be designed is given by

$$
\left(\begin{array}{l}
\dot{X} \\
\dot{Y} \\
\dot{Z}
\end{array}\right)=\left(\begin{array}{lll}
a_{11} & a_{12} & a_{13} \\
a_{21} & a_{22} & a_{23} \\
a_{31} & a_{32} & a_{33}
\end{array}\right)\left(\begin{array}{l}
X \\
Y \\
Z
\end{array}\right)=J\left(\begin{array}{l}
X \\
Y \\
Z
\end{array}\right),
$$

where $a_{11}=0.2857, a_{12}=10, a_{13}=0, a_{21}=0.1, a_{22}=-0.5, a_{23}=5, a_{31}=0, a_{32}=-15$, and $a_{33}=0$. There exists a unique equilibrium point $O(0,0,0)$ with the corresponding eigenvalues $\gamma=0.2896$ and $\sigma \pm j \omega=-0.2519 \pm j 8.5988$ due to the full rank of the Jacobin matrix of 
(2.2). The system (2.2) therefore possesses a saddle focus of index 1 satisfying the Shil'nikov inequality $|\gamma|>|\sigma|>0$. The corresponding eigenvectors are described by

$$
\begin{gathered}
\eta=\xi_{R} \pm j \xi_{I}=\left(\begin{array}{l}
\xi_{1 R} \\
\xi_{2 R} \\
\xi_{3 R}
\end{array}\right) \pm j\left(\begin{array}{l}
\xi_{1 I} \\
\xi_{2 I} \\
\xi_{3 I}
\end{array}\right)=\left(\begin{array}{c}
-0.4998 \\
0.0126 \\
0.7512
\end{array}\right) \pm j\left(\begin{array}{c}
0.0166 \\
0.4306 \\
0
\end{array}\right), \\
\mu=\xi_{\gamma}=\left(\begin{array}{l}
\xi_{1 \gamma} \\
\xi_{2 \gamma} \\
\xi_{3 \gamma}
\end{array}\right)=\left(\begin{array}{c}
0.9998 \\
0.0004 \\
-0.0199
\end{array}\right) .
\end{gathered}
$$

The 1-D unstable manifold $E_{\gamma}^{U}(O)$ corresponding to the real eigenvalue $\gamma=0.2896$ and the 2-D stable manifold $E_{\sigma+j \omega}^{S}(O)$ corresponding to the complex conjugate eigenvalues $\sigma \pm j \omega=-0.2519 \pm j 8.5988$ are given by

$$
\begin{gathered}
E_{\gamma}^{U}(O): \frac{X}{l}=\frac{Y}{m}=\frac{Z}{n}, \\
E_{\sigma+j \omega}^{S}(O): A X+B Y+C Z=0,
\end{gathered}
$$

where $(l, m, n)=(0.9998,0.0004,-0.0199)$, implying the direction of the 1-D unstable manifold, and $(A, B, C)=(0.3235,0.0125,0.2150)$, indicating the direction numbers of the 2-D stable manifold $E_{\sigma+j \omega}^{S}(O)$.

\section{Constructing a Switching Chaotic System with Saddle Foci of Index 1}

In this section, starting from the basic 3-D linear nominal system (2.2), and based on the heteroclinic Shil'nikov theorem and the switching control strategy, the necessary parameter conditions, which ensure the existence of the heteroclinic loop connecting two saddle foci of index 1 in the controlled switching system, are deduced. The intended 2-segmented piecewise linear controlled system which exhibits the chaotic behavior in the sense of the Smale horseshoe is then constructed.

According to the heteroclinic Shil'nikov theorem and the switching control strategy, a switching controller $\mathrm{F}(x, y, z)$ with $S_{Z}=\{(x, y, z) \mid z=0\}$ being the switching plane is introduced into the system (2.2). A coordinate transformation at the equilibrium point $O(0,0,0)$ is then performed by the switching controller $\mathbf{F}(x, y, z)$. As a result, one gets

$$
\left(\begin{array}{l}
\dot{x} \\
\dot{y} \\
\dot{z}
\end{array}\right)=\left(\begin{array}{lll}
a_{11} & a_{12} & a_{13} \\
a_{21} & a_{22} & a_{23} \\
a_{31} & a_{32} & a_{33}
\end{array}\right)\left(\left(\begin{array}{l}
x \\
y \\
z
\end{array}\right)-\mathbf{F}(x, y, z)\right),
$$




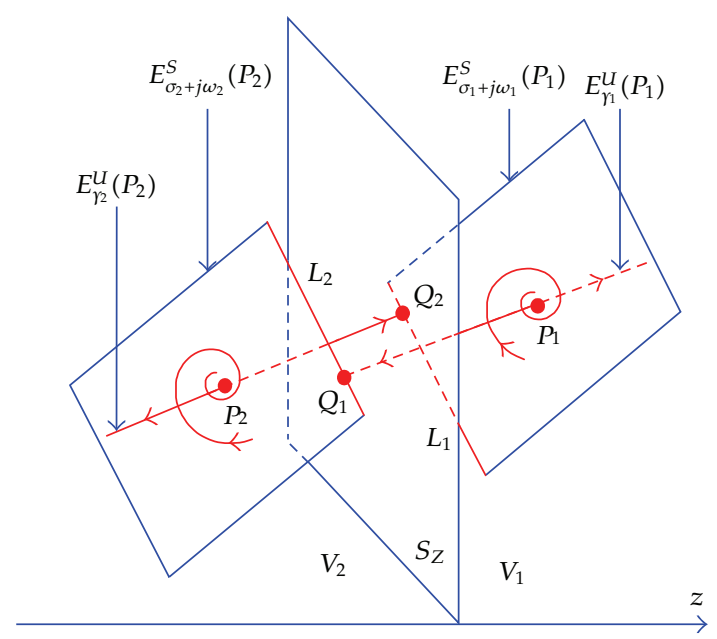

Figure 1: The eigen-subspace corresponding to $P_{1}$ and $P_{2}$.

where $a_{11}=0.2857, a_{12}=10, a_{13}=0, a_{21}=0.1, a_{22}=-0.5, a_{23}=5, a_{31}=0, a_{32}=-15$, and $a_{33}=0$.

Obviously, the system (3.1) possesses two equilibrium points $P_{1}\left(x_{1}, y_{1}, z_{1}\right) \in V_{1}$ and $P_{2}\left(x_{2}, y_{2}, z_{2}\right) \in V_{2}$ appearing to lie on the opposite sides of the switching plane $S_{Z}$. Note that the detailed mathematical expression of the switching controller $\mathbf{F}(x, y, z)=$ $\left[f_{1}(x, y, z), f_{2}(x, y, z), f_{3}(x, y, z)\right]^{T}$ in the system (3.1) will be determined by the necessary parameter conditions for forming a heteroclinic loop which connects $P_{1}$ and $P_{2}$, the two saddle foci of index 1 .

Let $E_{\gamma_{1}}^{U}\left(P_{1}\right)$ denote the one-dimensional unstable manifold of the equilibrium point $P_{1}$ corresponding to the real eigenvalue $\gamma_{1}=0.2896, E_{\gamma_{2}}^{U}\left(P_{2}\right)$ denote the one-dimensional unstable manifold of the equilibrium point $P_{2}$ corresponding to the real eigenvalue $\gamma_{2}=$ 0.2896, $E_{\sigma_{1}+j \omega_{1}}^{S}\left(P_{1}\right)$ denote the two-dimensional stable manifold of $P_{1}$ corresponding to the complex conjugate eigenvalues $\sigma_{1} \pm j \omega_{1}=-0.2519 \pm j 8.5988$, and $E_{\sigma_{2}+j \omega_{2}}^{S}\left(P_{2}\right)$ denote the two-dimensional stable manifold of $P_{2}$ corresponding to the complex conjugate eigenvalues $\sigma_{2} \pm j \omega_{2}=-0.2519 \pm j 8.5988$, respectively. Then, one gets

$$
\begin{gathered}
E_{\gamma_{i}}^{U}\left(P_{i}\right): \frac{x-x_{i}}{l}=\frac{y-y_{i}}{m}=\frac{z-z_{i}}{n}, \\
E_{\sigma_{i}+j \omega_{i}}^{S}\left(P_{i}\right): A\left(x-x_{i}\right)+B\left(y-y_{i}\right)+C\left(z-z_{i}\right)=0,
\end{gathered}
$$

where $i=1,2, l=0.9998, m=0.0004, n=-0.0199, A=0.3235, B=0.0125$, and $C=0.2150$. The eigen-subspace corresponding to $P_{1}$ and $P_{2}$ with orbits on the stable planes spirally approaching the equilibrium points is shown in Figure 1.

Let $Q_{1}$ be the cross point of $E_{\gamma_{1}}^{U}\left(P_{1}\right)$ and $S_{Z}, Q_{2}$ be the cross point of $E_{\gamma_{2}}^{U}\left(P_{2}\right)$ and $S_{Z}$, $E_{\sigma_{1}+j \omega_{1}}^{S}\left(P_{1}\right)$ and $S_{Z}$ intersect in the line $L_{1}$, and $E_{\sigma_{2}+j \omega_{2}}^{S}\left(P_{2}\right)$ and $S_{Z}$ intersect in the line $L_{2}$, 
respectively. Then, one gets

$$
\begin{gathered}
Q_{1}=E_{\gamma_{1}}^{U}\left(P_{1}\right) \cap S_{Z}:\left(x_{1}-\frac{l}{n} z_{1}, y_{1}-\frac{m}{n} z_{1}, 0\right), \\
Q_{2}=E_{\gamma_{2}}^{U}\left(P_{2}\right) \cap S_{Z}:\left(x_{2}-\frac{l}{n} z_{2}, y_{2}-\frac{m}{n} z_{2}, 0\right), \\
L_{1}=E_{\sigma_{1}+j \omega_{1}}^{S}\left(P_{1}\right) \cap S_{Z}:\left\{\begin{array}{l}
A\left(x-x_{1}\right)+B\left(y-y_{1}\right)+C\left(z-z_{1}\right)=0, \\
z=0,
\end{array}\right. \\
L_{2}=E_{\sigma_{2}+j \omega_{2}}^{S}\left(P_{2}\right) \cap S_{Z}:\left\{\begin{array}{l}
A\left(x-x_{2}\right)+B\left(y-y_{2}\right)+C\left(z-z_{2}\right)=0, \\
z=0 .
\end{array}\right.
\end{gathered}
$$

As indicated in Figure 1, there exists a heteroclinic orbit $H_{1}=E_{\gamma_{1}}^{U}\left(P_{1}\right) \cup Q_{1} \cup E_{\sigma_{2}+j \omega_{2}}^{S}\left(P_{2}\right)$ from $P_{1}$ to $P_{2}$ when $Q_{1}$ appears to lie on $L_{2}$. There exists another heteroclinic orbit $H_{2}=$ $E_{\gamma_{2}}^{U}\left(P_{2}\right) \cup Q_{2} \cup E_{\sigma_{1}+j \omega_{1}}^{S}\left(P_{1}\right)$ from $P_{2}$ to $P_{1}$ when $Q_{2}$ appears to lie on $L_{1}$. Provided that $Q_{1}$ appears to lie on $L_{2}$ and that $Q_{2}$ simultaneously appears to lie on $L_{1}$, there must exist a heteroclinic loop $H_{l}$ connecting $P_{1}$ and $P_{2}$ that is made up of the two heteroclinic orbits $H_{1}$ and $H_{2}$.

From (3.3), one gets that $Q_{1}$ appears to lie on $L_{2}$, and $Q_{2}$ simultaneously appears to lie on $L_{1}$ when the coordinate parameters of the equilibrium points $P_{1}\left(x_{1}, y_{1}, z_{1}\right) \in V_{1}$ and $P_{2}\left(x_{2}, y_{2}, z_{2}\right) \in V_{2}$ satisfy the following conditions:

$$
\begin{aligned}
& A\left(x_{1}-\frac{l}{n} z_{1}-x_{2}\right)+B\left(y_{1}-\frac{m}{n} z_{1}-y_{2}\right)-C z_{2}=0 \\
& A\left(x_{2}-\frac{l}{n} z_{2}-x_{1}\right)+B\left(y_{2}-\frac{m}{n} z_{2}-y_{1}\right)-C z_{1}=0
\end{aligned}
$$

According to the theory of the Smale horseshoe and the heteroclinic Shil'nikov theorem, when the system (3.1) is chaotic, there exist infinite numbers of heteroclinic orbits, among which there must exist a heteroclinic loop $H_{l}$ connecting $P_{1}$ and $P_{2}$ through $Q_{1}$ and $Q_{2}$. In this case, the system (3.1) exhibits the chaotic behavior in the sense of the Smale horseshoe.

In particular, when the switching plane is $S_{Z}=\{(x, y, z) \mid z=0\}$, and equilibrium points $P_{1}\left(x_{1}, y_{1}, z_{1}\right) \in V_{1}$ and $P_{2}\left(x_{2}, y_{2}, z_{2}\right) \in V_{2}$ are of certain symmetry, such as $x_{1}=-x_{2}=$ $x_{0}, y_{1}=y_{2}=y_{0} \geq 0$, and $z_{1}=-z_{2}=z_{0}>0$, (3.4) can be simplified into a single constraint equation, given by

$$
x_{0}=\frac{A l+B m-C n}{2 A n} z_{0}
$$


where $x_{0}$ exhibits dependence only on the value of $z_{0}$ and $y_{0}$ can be of arbitrary value. In brief, suppose that the equilibrium points $P_{1}\left(x_{1}, y_{1}, z_{1}\right) \in V_{1}$ and $P_{2}\left(x_{2}, y_{2}, z_{2}\right) \in V_{2}$ are appointed to satisfy the following coordinate parameter conditions:

$$
\begin{aligned}
& x_{1}=-x_{2}=\frac{z_{0}(A l+B m-C n)}{(2 A n)}, \\
& y_{1}=y_{2}, \\
& z_{1}=-z_{2}=z_{0},
\end{aligned}
$$

There exists a heteroclinic loop connecting equilibrium points $P_{1}$ and $P_{2}$ in the piecewise linear system (3.1).

For the sake of simplicity, let $y_{0}=0$ and $z_{0}=1$. From (3.6), one gets the coordinates of equilibrium points as: $P_{1}\left(x_{1}, y_{1}, z_{1}\right)=P_{1}(-25.4089,0,1)$ and $P_{2}\left(x_{2}, y_{2}, z_{2}\right)=P_{2}(25.4089,0,-1)$, which are the necessary parameter conditions for forming a heteroclinic loop. Based on the equilibrium points with the above-mentioned coordinates, one can further obtain the mathematical expression of the switching controller $\mathbf{F}(x, y, z)$ in the system (3.1), given by

$$
\mathbf{F}(x, y, z)=\left(\begin{array}{l}
f_{1}(x, y, z) \\
f_{2}(x, y, z) \\
f_{3}(x, y, z)
\end{array}\right)=\left(\begin{array}{c}
x_{0} \operatorname{sgn}(z) \\
y_{0} \operatorname{sgn}(z) \\
z_{0} \operatorname{sgn}(z)
\end{array}\right)
$$

where $x_{0}=-25.4089, y_{0}=0$, and $z_{0}=1$.

The numerical simulation results of the chaotic attractor, obtained from the piecewise linear system with saddle foci of index 1 described by (3.1) with (3.7), can be finally demonstrated as an illustrative example shown in Figure 2.

\section{Basic Dynamic Analysis of the Piecewise Linear Chaotic System}

In this section, a basic dynamic analysis of the piecewise linear chaotic system is given, including the dissipation, the Lyapunov exponents, and the solution of the state equations.

\subsection{Dissipation and Lyapunov Exponents}

According to (3.1) and (3.7), one can obtain the dissipation of the piecewise linear chaotic system, given by

$$
\nabla V=a_{11}+a_{22}+a_{33}=0.2857-0.5+0<0 .
$$

Therefore, the piecewise linear system with saddle foci of index 1 described by (3.1) with (3.7) is dissipative.

The Lyapunov exponents (LE) are also obtained to further prove the existence of chaos in the piecewise linear system, which are as follows: $\mathrm{LE}_{1}=0.27, \mathrm{LE}_{2}=0.00$, and $\mathrm{LE}_{3}=-0.49$. The existence of the positive LE implies the chaotic nature of the piecewise linear system. 


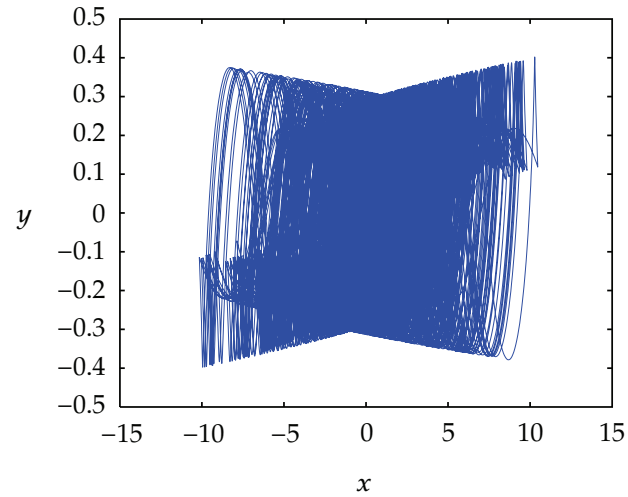

(a) $x$-y plane

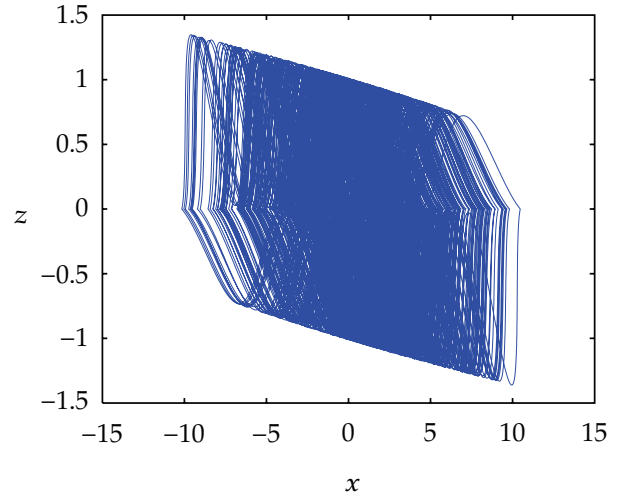

(b) $x$-z plane

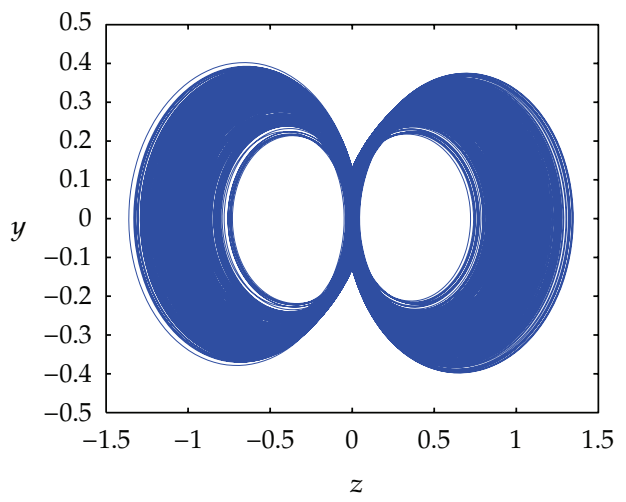

(c) $y$-z plane

Figure 2: Chaotic attractor obtained from the piecewise linear system with saddle foci of index 1.

\subsection{The Solution of the State Equations}

The solution $X(t)=(X(t), Y(t), Z(t))^{T}$ of the linear system (2.2) with the initial value $X(0)=$ $(X(0), Y(0), Z(0))^{T}$ is given by

$$
\mathbf{X}(t)=P\left(\begin{array}{ccc}
e^{\gamma t} & 0 & 0 \\
0 & e^{\sigma t} \cos \omega t & e^{\sigma t} \sin \omega t \\
0 & -e^{\sigma t} \sin \omega t & e^{\sigma t} \cos \omega t
\end{array}\right) P^{-1} \mathbf{X}(0)=\left(\begin{array}{lll}
b_{11} & b_{12} & b_{13} \\
b_{21} & b_{22} & b_{23} \\
b_{31} & b_{32} & b_{33}
\end{array}\right) \mathbf{X}(0)=B \mathbf{X}(0)
$$

where

$$
P=\left(\begin{array}{lll}
\xi_{1 \gamma} & \xi_{1 R} & \xi_{1 I} \\
\xi_{2 \gamma} & \xi_{2 R} & \xi_{2 I} \\
\xi_{3 \gamma} & \xi_{3 R} & \xi_{3 I}
\end{array}\right)
$$


From (2.3) and (4.3), the coefficients $b_{i j}(0 \leq i, j \leq 3)$ in (4.2) are given by

$$
\begin{aligned}
& b_{11}=1.0134 e^{\gamma t}-0.0134 e^{\sigma t} \cos \omega t-0.0013 e^{\sigma t} \sin \omega t, \\
& b_{21}=3.9007 \times 10^{-4} e^{\gamma t}-3.9007 \times 10^{-4} e^{\sigma t} \cos \omega t+0.0116 e^{\sigma t} \sin \omega t, \\
& b_{31}=-0.0202 e^{\gamma t}+0.0202 e^{\sigma t} \cos \omega t+0.0013 e^{\sigma t} \sin \omega t, \\
& b_{12}=0.0390 e^{\gamma t}-0.0390 e^{\sigma t} \cos \omega t+1.1605 e^{\sigma t} \sin \omega t, \\
& b_{22}=1.5014 \times 10^{-5} e^{\gamma t}+1.0000 e^{\sigma t} \cos \omega t-0.0289 e^{\sigma t} \sin \omega t, \\
& b_{32}=-7.7778 \times 10^{-4} e^{\gamma t}+7.7778 e^{\sigma t} \cos \omega t-1.7444 e^{\sigma t} \sin \omega t, \\
& b_{13}=0.6736 e^{\gamma t}-0.6736 e^{\sigma t} \cos \omega t-0.0424 e^{\sigma t} \sin \omega t, \\
& b_{23}=2.5926 \times 10^{-4} e^{\gamma t}-2.5926 \times 10^{-4} e^{\sigma t} \cos \omega t+0.5815 e^{\sigma t} \sin \omega t, \\
& b_{33}=-0.0134 e^{\gamma t}+1.0134 e^{\sigma t} \cos \omega t+0.0301 e^{\sigma t} \sin \omega t,
\end{aligned}
$$

where $\gamma=0.2896, \sigma=-0.2519$, and $\omega=8.5988$.

Since the piecewise linear chaotic system (3.1) is obtained from (2.2) by the coordinate transformation with the switching control strategy, the solution of (3.1) is obtained based on (4.2) as follows:

$$
\begin{gathered}
\mathbf{X}_{1}(t)=\mathbf{X}(t)-\mathbf{P}_{1} \quad \text { if } x>0, \\
\mathbf{X}_{1}(t)=\mathbf{X}(t)-\mathbf{P}_{2} \quad \text { if } x<0, \\
\dot{\mathbf{X}}_{1}(t)=B \mathbf{X}(t),
\end{gathered}
$$

where $\mathbf{X}_{1}(t)=(x(t), y(t), z(t))^{T}$ is the solution of the system (3.1), $\mathbf{X}(t)=(X(t), Y(t), Z(t))^{T}$ is the solution of the system $(2.2), \mathbf{X}(0)=(X(0), Y(0), Z(0))^{T}$ is the initial value, and $\mathbf{P}_{1}=$ $\left(x_{1}, y_{1}, z_{1}\right)^{T}$ and $\mathbf{P}_{2}=\left(x_{2}, y_{2}, z_{2}\right)^{T}$ are the equilibrium points.

\section{Conclusions}

Both saddle foci of index 1 and saddle foci of index 2 play a crucial role in chaos generation in 3-D autonomous systems, whenever they are smooth continuous chaotic systems or piecewise linear chaotic ones. Saddle foci of index 2 have been usually considered for creating double-scroll or double-wing chaotic attractors in systems, such as the double-scroll Chua system, the double-wing Lorenz system, and switching chaotic systems. Few detailed reports have addressed the issue of applying saddle foci of index 1 for chaos generation. To break such a limitation, this paper has proposed the application of saddle foci of index 1 for constructing chaotic systems, by using the switching control strategy and yielding a heteroclinic loop which connects two saddle foci of index 1 . On comparison with the existing methods, the approach presented in this paper is characterized mainly by selecting saddle foci of index 1 for chaos generation such that the intended 2-segmented piecewise linear system exhibits the chaotic behavior in the sense of the Smale horseshoe. Furthermore, this 
approach is more of generalization for constructing switching chaotic systems since the normal direction of uncontrolled basic 3-D linear system is arbitrarily orientated and is not supposed to be fixed on a special position.

\section{Acknowledgments}

This work was supported by the National Natural Science Foundation of China under Grants 60572073, 60871025 and 61172023, the Natural Science Foundation of Guangdong Province under Grants 8151009001000060, 8351009001000002, 9151009001000030, and S2011010001018, and the Science and Technology Program of Guangdong Province under Grant 2009B010800037.

\section{References}

[1] L. O. Chua, M. Komuro, and T. Matsumoto, "The double scroll family," IEEE Transactions on Circuits and Systems I, vol. 33, no. 11, pp. 1073-1117, 1986

[2] E. N. Lorenz, "Deterministic nonperiodic flow," Journal of the Atmospheric Sciences, vol. 20, pp. 130-141, 1963.

[3] G. R. Chen and T. Ueta, "Yet another chaotic attractor," International Journal of Bifurcation and Chaos, vol. 9, no. 7, pp. 1465-1466, 1999.

[4] J. C. Sprott, "A new class of chaotic circuit," Physics Letters A, vol. 266, no. 1, pp. 19-23, 2000.

[5] J. C. Sprott, "Simple chaotic systems and circuits," American Journal of Physics, vol. 68, no. 8, pp. 758$763,2000$.

[6] T. Zhou and G. Chen, "Classification of chaos in 3-D autonomous quadratic systems-I. Basic framework and methods," International Journal of Bifurcation and Chaos, vol. 16, no. 9, pp. 2459-2479, 2006.

[7] M. E. Yalçin, J. A. K. Suykens, J. Vandewalle, and S. Özouz, "Families of scroll grid attractors," International Journal of Bifurcation and Chaos, vol. 12, no. 1, pp. 23-41, 2002.

[8] G. Q. Zhong, K. F. Man, and G. Chen, "A systematic approach to generating n-scroll attractors," International Journal of Bifurcation and Chaos, vol. 12, no. 12, pp. 2907-2915, 2002.

[9] S. Yu, J. Lü, G. Chen, and X. Yu, "Design and implementation of grid multiwing butterfly chaotic attractors from a piecewise Lorenz system," IEEE Transactions on Circuits and Systems II, vol. 57, no. 10, Article ID 5575404, pp. 803-807, 2010.

[10] G. Li and X. Chen, "Constructing piecewise linear chaotic system based on the heteroclinic Shil'nikov theorem," Communications in Nonlinear Science and Numerical Simulation, vol. 14, no. 1, pp. 194-203, 2009.

[11] S. Yu, J. Lü, G. Chen, and X. Yu, "Generating grid multiwing chaotic attractors by constructing heteroclinic loops into switching systems," IEEE Transactions on Circuits and Systems II, vol. 58, no. 5, pp. 314-318, 2011.

[12] C. P. Silva, "Shil'nikov's theorem-a tutorial," IEEE Transactions on Circuits and Systems I, vol. 40, no. 10, pp. 675-682, 1993. 


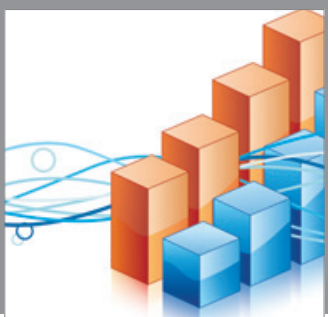

Advances in

Operations Research

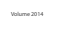

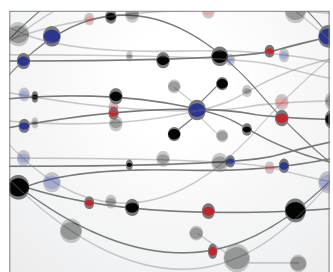

\section{The Scientific} World Journal
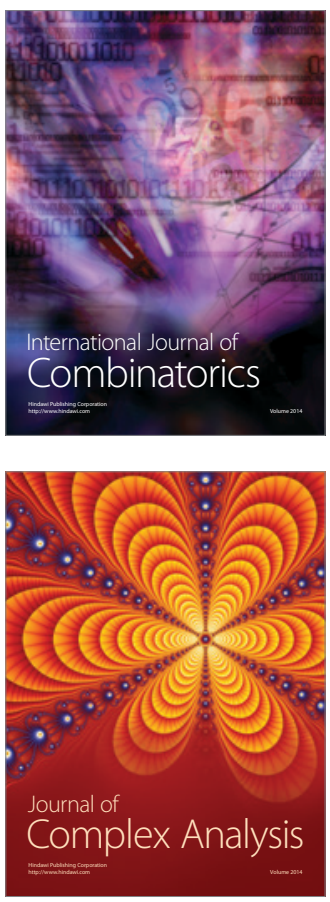

International Journal of

Mathematics and

Mathematical

Sciences
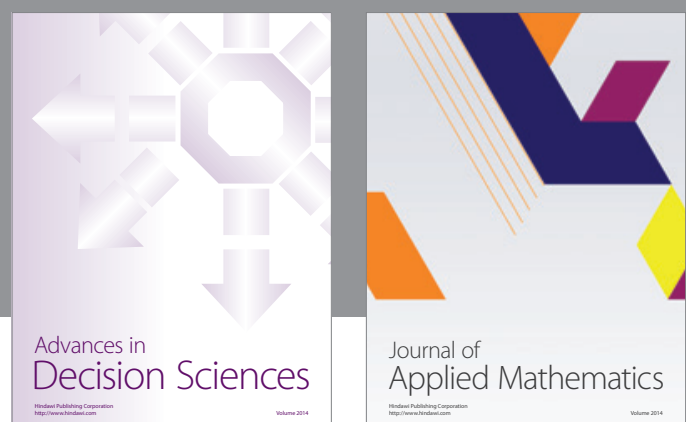

Journal of

Applied Mathematics
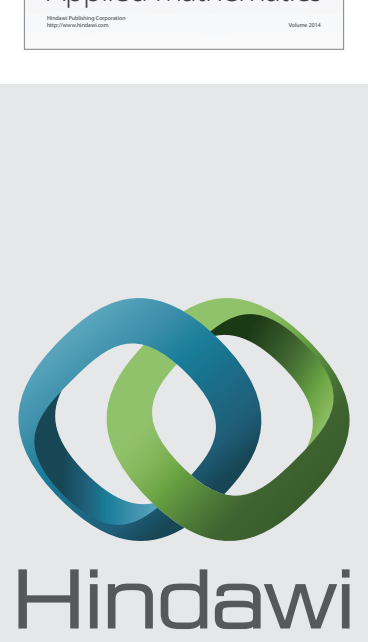

Submit your manuscripts at http://www.hindawi.com
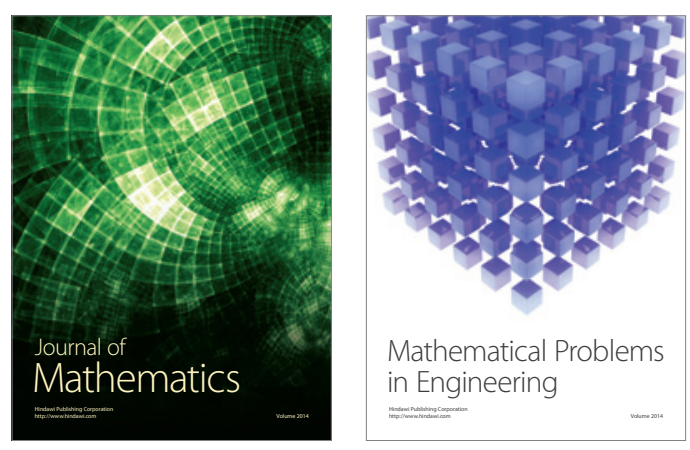

Mathematical Problems in Engineering
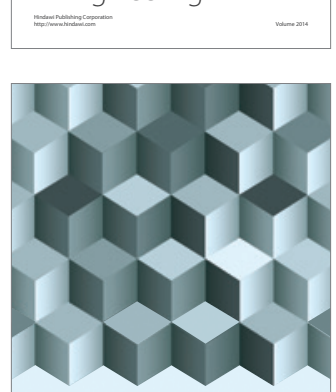

Journal of

Function Spaces
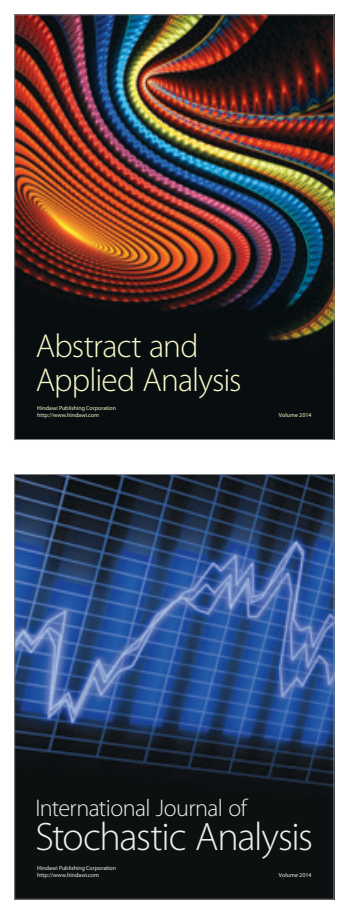

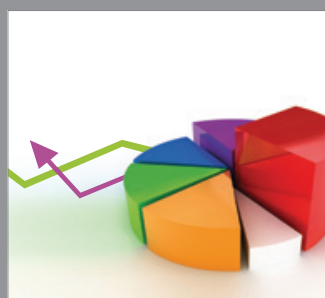

ournal of

Probability and Statistics

Promensencen
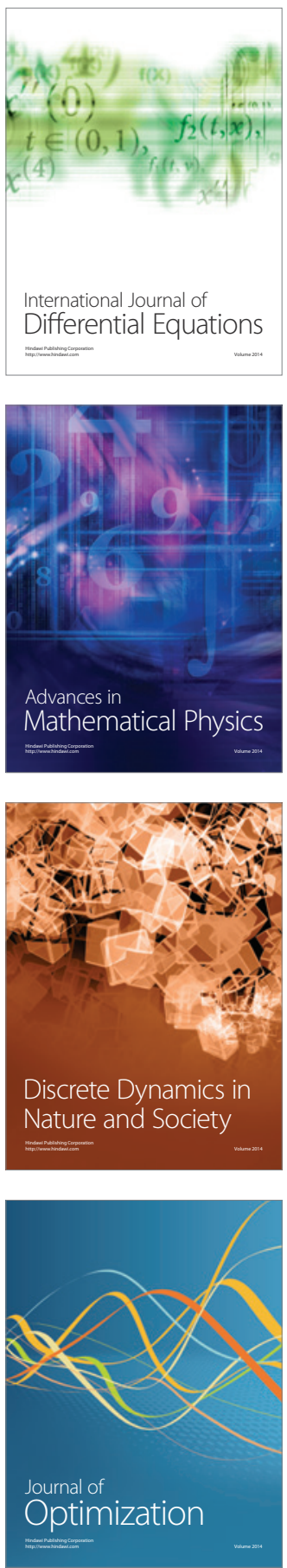